\title{
Correspondence
}

\section{Febrile convulsions and anticonvulsant} therapy

Sir,

Aldridge Smith and Wallace ${ }^{1}$ argue in favour of giving continuous anticonvulsant medication to prevent possible recurrences of febrile seizures. In our opinion, methodological errors make this conclusion questionable.

The assignment of treatment in this study was not only non-random, but was made in a manner that eliminates the possibility of making useful comparisons of Griffiths developmental quotients (GDQ). Specifically, all children of a younger age and abnormal neurodevelopment were given anticonvulsant medication. Others were assigned sequentially, but socioeconomic level influenced compliance if medication was assigned, ${ }^{2}$ and noncompliant patients were considered untreated. All of these factors affect significantly the outcome of developmental testing-for example, early infant testing depends heavily on evaluating motor function, while testing at a later age is based on verbal performance, which is closely related to socioeconomic level. The mere demonstration in Table $1^{1}$ that assignment groups did not differ greatly in GDQ scores at the start of treatment does not validate the eventual comparison at 24 months, if the patients' prognosis was confounded by their treatment.

In addition, the recurrence comparison (Table 3$)^{1}$ is invalid. Since anticonvulsant medication is known to reduce the rate of recurrence of fits, ${ }^{3}$ and the choice of treatment was based on factors which affect developmental scores, any comparison between these will reflect factors determining the choice of treatment rather than the possible effect of recurrent febrile seizures.

An evaluation of the effects of treatment and the recurrence of fits should begin with groups which are comparable with regard to important factors such as age, socioeconomic level, and prior neurological status. The proper comparison to be made is the change in GDQ over 24 months.

This paper does not, in our opinion, supply the clinician with an adequate basis on which to make decisions on treatment.

\section{References}

1 Aldridge Smith J, Wallace S J. Febrile convulsions: intellectual progress in relation to anticonvulsant therapy and to recurrence of fits. Arch Dis Child 1982; 57: 104-7.

2 Wallace S J. Prevention of recurrent febrile seizures using continuous prophylaxis: sodium valproate compared with phenobarbital. In: Nelson K B, Ellenberg $\mathbf{J} \mathbf{H}$, eds. Febrile seizures. New York: Raven Press, 1981: 135-42.

3 Wolf S M, Carr A, Davis D C, et al. The value of phenobarbital in the child who has had a single febrile seizure: a controlled prospective study. Pediatrics 1977; 59: 378-85.

$$
\begin{array}{r}
\text { D G HiRTz, Y J LEE, K B NeLson, } \\
\text { AND J H ElLENBERG } \\
\text { National Institute of Neurological } \\
\text { and Communicative Disorders and Stroke, } \\
\text { Bethesda, Maryland 20205, USA }
\end{array}
$$

Dr Aldridge Smith and Dr Wallace comment:

Age and neurological status were not as unevenly distributed between our groups as Hirtz et al. suggest. As we stated in our paper, 'Even among those considered at high risk of another convulsion, there were parents who were unwilling to give regular anticonvulsants. Thus the groups eventually receiving phenobarbitone, sodium valproate, or no treatment were comparable for factors considered to carry a risk of recurrence of seizures.' Those children with and without subsequent fits covered a comparable range of these factors. A direct comparison of GDQs at 24 months post initial fit (GDQ24), none the less, may be regarded as too blunt a test without an explicit control for other variables.

Our conclusions are, however, also confirmed when the comparisons are made using a regression analysis to control for age, social class, neurological status, sex, and initial GDQ.

$$
\begin{aligned}
& F=\frac{\mathbf{R}^{2} \mathrm{y} \text {. control }}{1-\mathbf{R}^{2} \mathrm{aU}} \times \frac{\mathrm{DFe}}{\overline{D f y}} \text { where } \mathrm{R}^{2} \mathrm{y} \text {. control }= \\
& \mathbf{R}^{2} \mathrm{aU}-\mathbf{R}^{2} \text { control, } \mathbf{R}^{2} \mathrm{e}=1-\mathbf{R}^{2} \mathrm{aU} \text {, and } \mathrm{DFe}=\mathrm{N}-\mathrm{K}-1 .
\end{aligned}
$$

The F-value of the comparison of GDQ24 for children without subsequent fits and in receipt of continuous medication (drug present in the blood at each of the five times of testing) or who continuously received no drug is not significant $(F=0.0013 / 0.2371 \times 42 / 1=$ $0 \cdot 241, \mathrm{P}>0.05$ ). Comparison between all those children without subsequent fits and all those with subsequent fits shows the negative effect of subsequent fits on GDQ24 to be significant at the $5 \%$ level $(F=0.0110 /$ $0.2307 \times 107 / 1=5.120, P<0.05)$. The effect of the number of subsequent fits from $(0$ to 7$)$ is significant at the $1 \%$ level $(F=0.0169 / 0.2248 \times 107 / 1=8.058$, $\mathbf{P}<0.01)$

We think these results confirm that our study does supply the clinician with an adequate basis for making treatment decisions. 'For children at high risk the present study supports continuous anticonvulsant medication until the child is past the vulnerable age. For those at low risk the present evidence suggests that treatment without the use of anticonvulsants should be considered.'

\section{Plasma alkaline phosphatase activity in rickets of immaturity}

Sir,

We were interested to read the article by Glass et al. ${ }^{1}$ In a similar study ${ }^{2}$ we showed that in a group of 26 
non-rachitic infants, 14 (54\%) showed an increase-peakdecrease pattern of bone origin alkaline phosphatase activity with increasing postconceptional age. There was no significant change in plasma calcium or inorganic phosphorus.

Because of the difference in techniques of estimation it is not possible to compare directly our published results with those of Glass et al. Their recommendation of $500 \mathrm{U} / 1$ as an upper limit of normal cannot be directly related to our laboratory, or to any which used different methodology.

It is also difficult to define age based reference intervals for plasma alkaline phosphatase activity in preterm infants because of the non-Gaussian distribution of data. Moreover, because infants of identical postnatal age, but differing postconceptional ages, are not equivalent, it is not appropriate to use postnatal age as a basis for establishing normal values. We have noticed that alkaline phosphatase activity when measured serially in an infant will invariably exceed, at some stage, a gestational age related range (I Kovar et al., unpublished data).

To avoid these problems we suggest that alkaline phosphatase activity in an individual neonate and infant should be expressed in relation to a fixed range, rather than as a gestation related mean and standard deviation; use of a percentage of an upper limit of an adult reference range permits comparison between laboratories. This then allows recommendations to be made for intervention in a given case when a fixed multiple is recorded. We agree, however, that serial plasma alkaline phosphatase activity estimation is a useful means of screening for rickets in this susceptible population.

\section{References}

1 Glass E J, Hume R, Hendry G M A, Strange R C, Forfar J O. Plasma alkaline phosphatase activity in rickets of prematurity. Arch Dis Child 1982; 57: 373-6.

2 Kovar I, Mayne P, Barltrop D. Plasma alkaline phosphatase activity: a screening test for rickets in preterm neonates. Lancet 1982 ; i: 308-10.

ILYA Kovar AND DONALD BARLTROP Department of Child Health, Westminster Medical School, Westminster Children's Hospital, Vincent Square, London SWIP 2NS

PhiLIP MAYNe

Department of Chemical Pathology,

The Royal Free Hospital, London NW3 $2 Q G$

\section{Skeletal changes in preterm infants}

Sir,

We were interested to read the paper by Koo et al. ${ }^{1}$ in which they mention that none of the infants fed exclusively on breast milk had abnormal skeletal $x$-rays.

We undertook a similar study of the possible effect of breast milk or humanised formula on infants' blood 25-hydroxycholecalciferol and parathyroid hormone concentrations. ${ }^{2}$ It was found that parathyroid hormone concentrations at age 8 weeks (measured by radioimmunoassay which predominantly detects the $\mathrm{C}$ terminal end) were normal in infants fed exclusively on breast milk, but were appreciably higher in infants receiving a formula milk in addition to breast milk This difference was not explained by the plasma 25hydroxycholecalciferol concentrations, which were normal and similar in both groups. We wonder whether the serum biochemical findings (calcium, phosphate, alkaline phosphatase, immunoreactive parathyroid hormone) and radiological findings in the 7 infants fed exclusively on breast milk were different from those of the 7 infants fed with the formula in the study of Koo et al.

\section{References}

1 Koo W W K, Gupta J M, Nayanar V V, Wilkinson M, Posen S. Skeletal changes in preterm infants. Arch Dis Child 1982; 57: 447-52.

2 Sann L, Rigal D, David L, Frederich A, Lahet C. Late evolution of serum immunoreactive parathyroid hormone, calcitonin, and plasma 25-hydroxycholecalciferol concentrations in very low birthweight infants. Acta Paediat Scand 1981; 70: 479-84.

LANN

Hopital Debrousse, 29 rue Saur-Bouvier 69322 Lyon

Cedex 05,

France

\section{Dr Koo and co-workers comment;}

We have reviewed the data from our study ${ }^{1}$ and those published by Sann et al. ${ }^{2}$ It appears there are a number of aspects which make direct comparison of the results difficult. The patients in our study who developed radiological skeletal abnormalities have more severe illnesses and have significantly lower birthweights than those with normal skeletal $x$-rays. In addition, 9 infants in our study received human milk for 2 to 37 (median 18) days-a period far shorter than the formula supplemented group of infants in the study of Sann et al. ${ }^{2}$ Furthermore, the dose of vitamin $\mathrm{D}_{2}$ supplement used in our study was $800 \mathrm{IU} /$ day from age 14 days compared with the dose of $2400 \mathrm{IU} /$ day from age 10 days in the study of Sann et $a .^{2}$

However, we analysed our data further, using one way analysis of variance and Newman Keuls' multiple comparison procedure to determine if there were any differences between the infants with radiological skeletal abnormalities (group 1) and the infants with normal skeletal radiographs but who were fed human milk only (group 2) and those who were fed some or no human milk (group 3).

The results showed that infants in group 2 were significantly larger (birthweight $1183 \pm 76 \mathrm{~g}$, mean $\pm 1 \mathrm{SD}$ ) and had greater gestational ages $(30 \pm 2.9$ weeks, mean \pm $1 \mathrm{SD})$ at birth than group 1 infants $(P<0.05)$. They also tolerated a predetermined volume of feeds $(>160 \mathrm{ml} / \mathrm{kg}$ per day) significantly sooner $(7 \cdot 3 \pm 1.9$ days of age, mean \pm 1 SD) than group 1 infants $(P<0.05)$. There were no significant differences in these parameters between groups 2 and 3 or between groups 1 and 3 .

There were no important intergroup differences in serum calcium, phosphorus, alkaline phosphatase, total protein, 25 hydroxyvitamin D (25-OHD), and immunoreactive parathyroid hormone in the cord blood, at 5 weeks and 10 weeks postnatal age. In group 2 infants, 